\title{
Genome-wide analysis of the interaction between the endosymbiotic bacterium Wolbachia and its Drosophila host Zhiyong Xi ${ }^{\dagger 1,2}$, Laurent Gavotte ${ }^{\dagger 1,3}$, Yan Xie ${ }^{4}$ and Stephen L Dobson*1
}

Address: ${ }^{1}$ Department of Entomology; University of Kentucky; Lexington, KY 40546, USA, ${ }^{2}$ Department of Entomology, Michigan State University; East Lansing, MI 48824, USA, ${ }^{3}$ Institut des Sciences de l'Evolution. UMR-CNRS 5554, Bat 24, CC063. Université Montpellier II. Place Eugène Bataillon 34095 Montpellier Cedex 5, France and ${ }^{4}$ Department of Statistics, University of Kentucky, Lexington, KY 40546, USA

Email: Zhiyong Xi - xizy@msu.edu; Laurent Gavotte - Laurent.gavotte@univ-montp2.fr; Yan Xie - Yan.Xie@sanofipasteur.com; Stephen L Dobson* - sdobson@email.uky.edu

* Corresponding author †Equal contributors

Published: 2 january 2008

BMC Genomics 2008, 9:1 doi:10.1/86/147|-2164-9-I
Received: 16 August 2007

Accepted: 2 January 2008

This article is available from: http://www.biomedcentral.com/I47I-2164/9/I

(C) 2008 Xi et al; licensee BioMed Central Ltd.

This is an Open Access article distributed under the terms of the Creative Commons Attribution License (http://creativecommons.org/licenses/by/2.0), which permits unrestricted use, distribution, and reproduction in any medium, provided the original work is properly cited.

\begin{abstract}
Background: Intracellular Wolbachia bacteria are obligate, maternally-inherited, endosymbionts found frequently in insects and other invertebrates. The success of Wolbachia can be attributed in part to an ability to alter host reproduction via mechanisms including cytoplasmic incompatibility $(\mathrm{Cl})$, parthenogenesis, feminization and male killing. Despite substantial scientific effort, the molecular mechanisms underlying the Wolbachia/host interaction are unknown.
\end{abstract}

Results: Here, an in vitro Wolbachia infection was generated in the Drosophila S2 cell line, and transcription profiles of infected and uninfected cells were compared by microarray. Differentiallyexpressed patterns related to reproduction, immune response and heat stress response are observed, including multiple genes that have been previously reported to be involved in the Wolbachia/host interaction. Subsequent in vivo characterization of differentially-expressed products in gonads demonstrates that Angiotensin Converting Enzyme (Ance) varies between Wolbachia infected and uninfected flies and that the variation occurs in a sex-specific manner. Consistent with expectations for the conserved $\mathrm{Cl}$ mechanism, the observed Ance expression pattern is repeatable in different Drosophila species and with different Wolbachia types. To examine Ance involvement in the $\mathrm{Cl}$ phenotype, compatible and incompatible crosses of Ance mutant flies were conducted. Significant differences are observed in the egg hatch rate resulting from incompatible crosses, providing support for additional experiments examining for an interaction of Ance with the $\mathrm{Cl}$ mechanism.

Conclusion: Wolbachia infection is shown to affect the expression of multiple host genes, including Ance. Evidence for potential Ance involvement in the $\mathrm{Cl}$ mechanism is described, including the prior report of Ance in spermatid differentiation, Wolbachia-induced sex-specific effects on Ance expression and an Ance mutation effect on $\mathrm{Cl}$ levels. The results support the use of Wolbachia infected cell cultures as an appropriate model for predicting in vivo host/Wolbachia interactions. 


\section{Background}

Maternally transmitted Wolbachia are alpha proteobacteria that infect a wide range of invertebrates, including nematodes, mites, spiders, and an estimated $>20 \%$ of insect species $[1,2]$. The ability of Wolbachia infections to spread and persist within this broad range of hosts can be attributed in part to its induction of multiple reproductive abnormalities including cytoplasmic incompatibility (CI), parthenogenesis, feminization and male killing. CI is the most commonly reported phenotype and induces developmental arrest of embryos resulting from matings between females and males that are infected with different Wolbachia types $[3,4]$. There is substantial interest in defining the molecular basis of the Wolbachia/host interaction, including the mechanisms of Wolbachia intracellular maintenance within host cells and mechanisms by which Wolbachia manipulate host reproduction $[5,6]$. In addition to basic scientific interest, applied researchers are also interested in Wolbachia as a potential tool for novel applied strategies including population replacement and suppression $[7,8]$.

Similar to Rickettsia prowazekii, the Wolbachia genome contains genes encoding components of the Type IV secretion system (T4SS) [5,9], a pathogenic bacterial protein secretary pathway known to secrete various effector molecules affecting cell host physiology [10]. Presence of T4SS in Wolbachia genome suggests a potential alteration of host cell expression as a means of facilitating its intracellular survival and dissemination, as observed for other intracellular bacteria [11]. However, characterization of Wolbachia interaction in vivo is complicated by dynamic infection levels that are affected by host genotype and nutrition, variable tissue tropism, and Wolbachia expression patterns that differ with host age $[12,13]$. Thus, a simplified model system, such as an in vitro Wolbachia infection within a well characterized cell culture, could potentially provide a useful tool for studying mechanisms of the Wolbachia/host interaction.

Drosophila S2 cells are derived from embryonic phagocytic cells [14] and previously have been demonstrated to serve as a valid in vitro model for examining intracellular infections and as a system for gene expression studies using microarrays and RNAi technology [15-19]. In addition to characterizing cross talk between Wolbachia and host cells, in vitro Wolbachia infections are also being used for screens to identify novel drugs that impact obligate Wolbachia infections within medically important filarial nematodes [20-22]. The latter studies will benefit from validation of the in vitro system as a predictor of in vivo events and from an improved understanding of the Wolbachia/host interaction in vitro.
Here, we used Wolbachia infected S2 cells as a model system for studying the molecular mechanisms that determine the Wolbachia/host interaction. Initially, microarrays were used to examine for differential expression between uninfected and Wolbachia infected S2 cell cultures. To determine the utility of the $\mathrm{S} 2$ system as a predictor of in vivo differential expression, one differentially expressed transcript (Angiotensin converting enzyme; Ance) was subsequently examined in testes and ovaries of D. simulans and D. melanogaster. Ance acts as a peptidyldipeptidase or endopeptidase removing the C-terminal peptide from its substrate and is required for spermatogenesis in Drosophila [23]. Quantitative Reverse Transcriptase PCR (qRT-PCR) indicate that Ance is differentially expressed in infected and uninfected flies, consistent with results in the S2 in vitro system. Significantly higher Ance expression is observed in Wolbachiainfected ovaries relative to uninfected ovaries. In contrast, lower expression is observed in infected testes relative to uninfected testes.

Ance mutant flies were used to examine for potential involvement of Ance in the CI phenotype. The Ance mutant fly, designated l(2)34Eb2, was derived from EMS mutagenesis. Since both homozygotes and hemizygotes (34Eb2 and a deficiency) are male sterile [23,24], heterozygotes were employed in crosses. Comparison of crosses using Ance mutant flies and wild type flies revealed a significant difference in the egg hatch rate, consistent with a hypothesized interaction between Ance and the CI phenotype.

In addition to Ance, differential expression is observed with multiple transcripts related to sexual reproduction, immune response and heat stress response. The latter include genes that have been previously reported to be involved in the Wolbachia/host interaction. The results are discussed as a validation of the in vitro model and as support for examining additional genes which have been identified as variable between infected and uninfected cells.

\section{Results \\ Drosophila S2 cell line infection}

Quantitative PCR was used to measure the relative infection level in the $S 2_{I}$ cell line following an initial shell vial introduction (Fig. 1). After the first Wolbachia introduction, the infection level in the cell line was about $10^{5}$ times lower than in the DSR adult female. Therefore, the shell vial introduction was repeated sequentially as shown in Figure 1. Following five sequential Wolbachia introductions, the resulting Wolbachia infection level was increased 5.6 fold in the $\mathrm{S} 2_{\mathrm{I}}$ cell line. To generate the uninfected $\mathrm{S} 2_{\mathrm{U}}$ line, the $S 2_{\mathrm{I}}$ cell line was split and treated with tetracycline (Fig. 1). For transcriptomic comparison, the $\mathrm{S}_{\mathrm{U}}$ cell line 


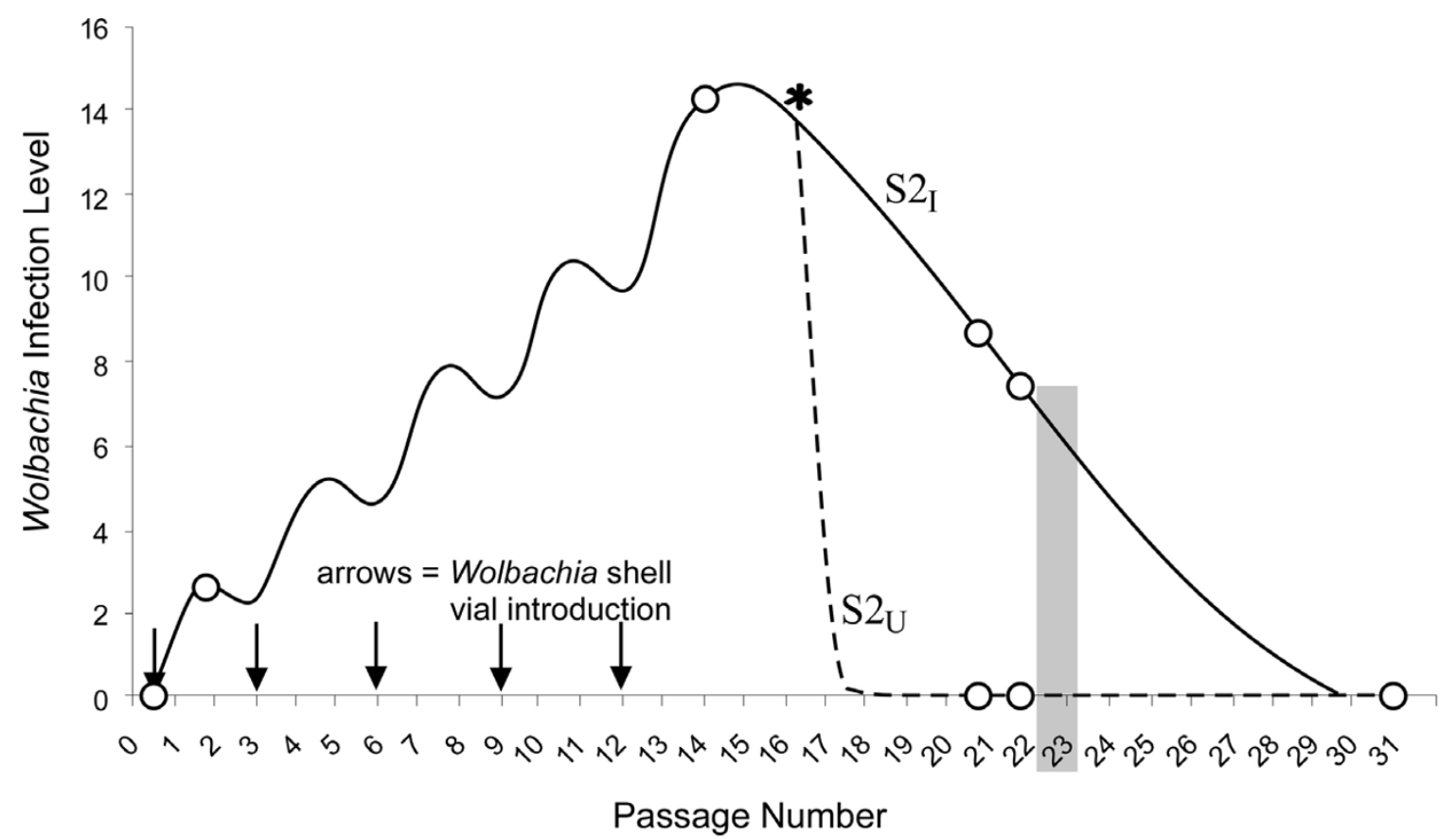

Figure I

Diagram illustrating the in vitro infection strategy, tetracycline clearing and microarray analysis of infected (S2 $)$ and uninfected $\left(\mathrm{S} 2_{U}\right)$ cell lines. The line illustrates an estimated infection level based upon periodic qPCR assays (indicated by hollow circles). The number provides a relative measure, not the absolute number of Wolbachia. The "waves" in the left half of the graph resulted from removal of the infection by host and enrichment of infection by shell vial introduction. Arrows indicate shell vial introduction of Wolbachia infection. At passage 17 (marked with asterisk), the cell line was split and one subline was tetracycline treated. At passage 23 (grey shading) the cell line was examined via microarray.

was used instead of the naive S2 line due to concerns that the genetic background of the S2 cell line might have been altered during the repeated transfection procedure. To avoid the potential complication caused by transcriptional differences induced by the tetracycline treatment, the microarray analysis was not conducted until six passages after antibiotic treatment. The absence of Wolbachia infection in the $S 2_{\mathrm{U}}$ line was confirmed by PCR with $w s p$ primers (440F/691R) at passages 21 and 22 (Fig 1).

\section{Differential in vitro expression}

The Genechip contains 13,966 probe sets, of which 7,197 were found to be uninformative (signal was absent in all eight replicates). The frequency of uninformative probes is not unexpected considering the use of cells derived from embryos (Genechip includes development stage and sexspecific probe sets) and results of prior studies (use of the same platform results in 4992 probe sets with signals below the detectable threshold; [25]). Of the remaining probes, 263 sets were different between S2 $2_{\mathrm{I}}$ and $\mathrm{S} 2_{\mathrm{U}}$ cells ( $\mathrm{P}<0.05$ and $\geq 1.2$ fold change) (Fig. 2). A complete set of microarray data results is available at Gene Expression Omnibus [26].
To facilitate integration of the results with currently available information about the Wolbachia/Drosophila interaction, transcripts are described relative to the biological process using Gene Ontology (GO) analysis. Of the differentially transcribed probe sets, fifty are categorized as genes with unknown functions and are not discussed subsequently. The remaining 213 probe sets are identified as belonging to a particular biological process ontology, with 73 down-regulated and 140 up-regulated. After all the differentially expressed genes were enriched into GO terms, the GO terms were ranked based on $\mathrm{Z}$ value. The top five non-redundant Gene Ontology (GO) terms are shown in Table 1. A complete set of GO data is available in the support information (Additional File 1 and 2).

\section{In vivo characterization of angiotensin converting enzyme (Ance)}

Among the probe sets on the chip, 153728_at (Ance) is the only gene that is differentially transcribed at the Bonferroni corrected level (Fig. 2). Therefore, Ance was selected for in vivo expression characterization. qRT-PCR was used to assay Ance mRNA levels in D. simulans and D. melanogaster ovaries and testis dissected from infected and uninfected flies. As shown in Figure 3, the average Ance 


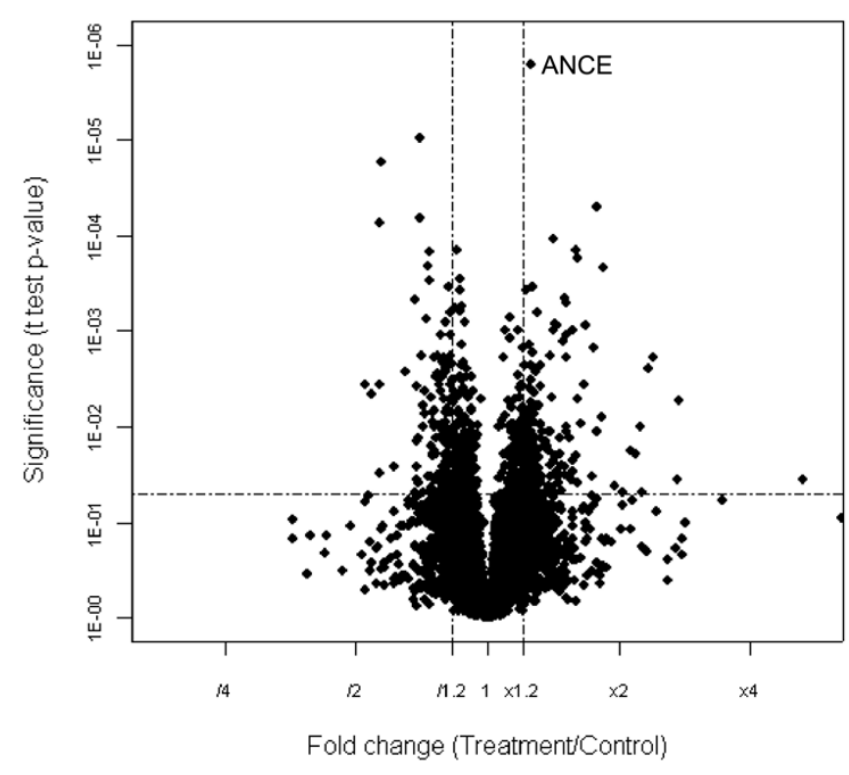

Figure 2

Distribution of the relative expression of 6,769 genes in the infected S2, versus uninfected $\mathrm{S}_{U}$ cell lines. Genes with differential expression are defined as those with $\mathrm{P}<0.05$ and a \pm 1.2 fold change.

expression is 2.1 fold higher in infected $D$. simulans ovaries relative to uninfected ovaries. In contrast, Ance expression was 1.5 fold lower in testes of Wolbachia infected $D$. simulans flies relative to infected testes. Similar results are observed with both the wild type (DMC) and the Ance mutant D. melanogaster flies (Fig. 3).

To examine for an interaction of Ance and Wolbachiainduced CI, crosses were conducted between wild type and Ance-mutant D. melanogaster strains that were either infected or uninfected. As shown in Table 2, no significant difference in hatch rate was observed in comparisons of the eight compatible cross types. In contrast, a comparison of the incompatible crosses revealed a higher egg hatch rate in crosses of infected wild-type males mated with uninfected Ance mutant females (40\% hatch) relative to other incompatible crosses (26-30\% hatch).

\section{Additional differentially-expressed transcripts}

In addition to Ance, microarray analysis revealed nineteen differentially transcribed probe sets representing genes previously reported to be associated with reproduction (Table 3). Among these, the majority function in the different process of oogenesis, including ovarian follicle cell development, pole plasm assembly and nurse cell to oocyte cell transport. l(2) $g l$, which has been recently hypothesized as involved in the CI mechanism [6], is also up-regulated. Enrichment and sorting of the differentiallyexpressed probe sets into their corresponding GO term with GeneFinder revealed "response to unfolded proteins" to be the term most frequently down-regulated (Table 1). From a total of 27 heat shock proteins in Drosophila, eleven (41\%) were lower in the Wolbachia infected cell culture. Up-regulation was not observed in this category (Fig. 4). "Antimicrobial humoral response" was identified as the most common up-regulated GO term following enrichment and sorting (Table 1). As shown in Figure 5, most of the genes in this GO term play a role in the immune signaling pathway, including Toll and Imd. Among these, two important regulatory proteins: Relish (Rel) and Dorsal $(D l)$, belong to the NF- $\kappa B$ family, and higher expression was observed in Wolbachia infected S2 cells. Five downstream antimicrobial peptides were also up-regulated, including attacin (A, B, C and D) and diptericin B (Fig. 5). In contrast, ird5 and peroxiredoxin 2540 were down-regulated.

Table I: The top five non-redundant Gene Ontology (GO) terms in biological process which contain the most differentially expressed genes. Up or down, up- or down-regulation after infection, respectively; $T$, number of genes assigned to this the $G O$ term; $M$, the subset of $T$ genes that are represented on the microarray; $C$, the subset of $M$ genes observed to vary in the analysis; $Z$, significance score

\begin{tabular}{|c|c|c|c|c|c|}
\hline & GO Term & $\mathrm{T}$ & M & C & Z \\
\hline \multirow[t]{5}{*}{ Up } & Antimicrobial humoral response (sensu Protostomia) & 70 & 20 & 6 & 7.4 \\
\hline & Negative regulation of cell proliferation & 42 & 10 & 3 & 5.3 \\
\hline & lon homeostasis & 40 & 10 & 3 & 5.3 \\
\hline & Larvae development (sensu Insecta) & 54 & 14 & 3 & 4.3 \\
\hline & Dorsal appendage formation & 39 & 15 & 3 & 4.1 \\
\hline \multirow[t]{5}{*}{ Down } & Response to unfolded protein & 50 & 15 & 6 & 12.6 \\
\hline & Response to chemical stimulus & 248 & 77 & 6 & 4.7 \\
\hline & Tissue morphogenesis & 78 & 16 & 2 & 3.7 \\
\hline & RNA splicing & 101 & 40 & 3 & 3.2 \\
\hline & Positive regulation of transcription, DNA-dependent & 102 & 34 & 2 & 2.2 \\
\hline
\end{tabular}




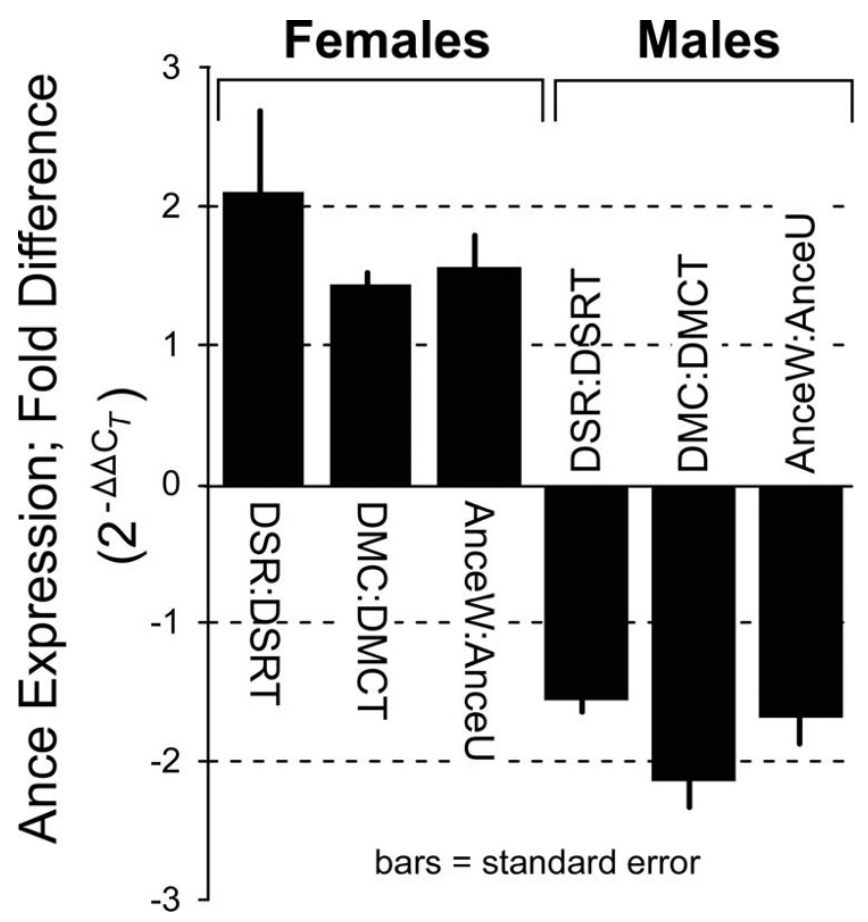

Figure 3

In vivo qRT-PCR assay of differential Ance expression in Wolbachia infected and uninfected $D$. simulans (DSR:DSRT), D. melanogaster wild type (DMC:DMCT) and D. melanogaster Ance mutant (AnceW:AnceU) flies. Wolbachia infection is consistently associated with higher Ance expression in ovaries and lower Ance expression in testes.

\section{Discussion}

A genome-wide analysis of the Wolbachia/host interaction has been conducted in vitro using the Drosophila S2 cell line. As a simplified model system, the results are likely to under-represent in vivo events (Type II error), for reasons including a relatively low Wolbachia infection level and the reduced complexity of the embryonic cell environment in the S2 cell line. However, the use of a simplified system is not an inappropriate approach given the dearth of information currently available for the Wolbachia-host interaction. An important motivation for subsequent in vivo transcriptional assays was to address the concern that the microarray results may represent an experimental artifact. Specifically, in vivo characterization of Ance expression via qRT-PCR was used to examine the validity of the in vitro system as a predictor of Ance in vivo expression. The selection of Ance was based primarily upon the observed level of differential expression and prior studies describing the potential involvement of Ance in spermatid differentiation [23], suggesting a possible role for Wolbachia/ Ance interaction. Specifically, Wolbachia has been observed to be associated with differentiating sperm [27], providing a potential role of sperm modification in CI $[28,29]$.

The location of Ance mutation has been mapped to 34E3-5 on the second chromosome [24]. The recessive lethality associate with the mutation due to the loss of function could be rescued by expression of Ance in the Ptransformation assay and genetic complementation test [24]. Homozygote Ance males are sterile. Their testes lack individualized sperm and have very few actin-based individualization complexes $[23,50]$. Therefore, premised upon the described involvement of Ance in spermatid differentiation, additional experiments were conducted to examine a model in which Wolbachia modifies the sperm via a pathway involving Ance expression. The latter experiments included characterization of Ance mRNA levels in testes and ovaries and examining egg hatch resulting from CI crosses of Ance flies.

Table 2: Cl Cross Assay Results

\begin{tabular}{|c|c|c|c|c|}
\hline Expected $\mathrm{Cl}$ Type & Cross * & Percent Egg Hatch † & Egg Counted & ANOVA** \\
\hline \multirow[t]{8}{*}{ Compatible } & $\mathrm{DMCT} \times \mathrm{DMCT}$ & $68.6 \pm 5.3 \% ; 13$ & 197 & a \\
\hline & $\mathrm{DMC} \times \mathrm{DMC}$ & $66.4 \pm 2.1 \% ; 38$ & 595 & \\
\hline & AnceU $\times$ AnceU & $73.8 \pm 3.1 \% ; 10$ & 248 & \\
\hline & AnceW $\times$ AnceW & $76.1 \pm 3.4 \% ; 10$ & 184 & \\
\hline & AnceW × DMC & $71.7 \pm 1.9 \% ; 61$ & 1173 & \\
\hline & $\mathrm{DMC} \times \mathrm{DMCT}$ & $66.7 \pm 4.3 \% ; 15$ & 168 & \\
\hline & DMC $\times$ AnceW & $66.9 \pm 4.0 \% ; 9$ & 118 & \\
\hline & $\mathrm{DMC} \times$ AnceU & $61.5 \pm 1.5 \% ; 10$ & 127 & \\
\hline \multirow[t]{4}{*}{ Incompatible } & $\mathrm{DMCT} \times \mathrm{DMC}$ & $28.4 \pm 1.8 \% ; 39$ & 440 & $b$ \\
\hline & DMCT $\times$ AnceW & $30.4 \pm 2.1 \% ; 40$ & 529 & $b$ \\
\hline & AnceU × DMC & $40.1 \pm 3.4 \% ; 46$ & 928 & c \\
\hline & AnceU $\times$ AnceW & $26.4 \pm 1.4 \% ; 30$ & 795 & b \\
\hline
\end{tabular}

*Female $\times$ Male

† Average \pm Standard Error; Number of Crosses

** Hatch rate was subjected to arcsine transformation before performing ANOVA, $P<0.05$ 


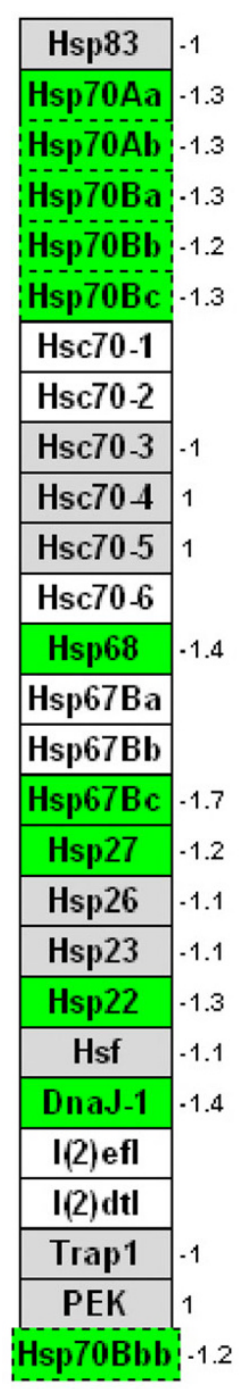

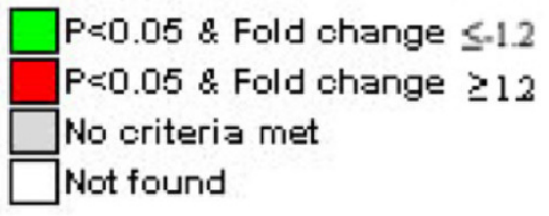

Figure 4

Regulation pattern of heat shock protein $(H s p)$ in Wolbachia infected S2 cells. All heat shock proteins in Drosophila are shown. Down-regulation (green) occurs in $41 \%$ (II of 27) of known Hsps. The fold change is shown for each gene.

The qRT-PCR assays demonstrate higher Ance expression in infected ovaries relative to uninfected ovaries. The latter observation is consistent with the higher Ance expression that is observed in the Wolbachia infected S2 cell line, which was derived from embryos. Future experiments should address whether the observed differential Ance expression originates within oocytes, maternally-derived ovary tissues, or both. Wolbachia infection was also associated with differential Ance expression in testes, but the variation was the reverse of that observed in ovaries. Lower Ance expression was observed in Wolbachiainfected testes relative to testes from uninfected flies. A similar pattern is observed in D. simulans and D. mela- nogaster. It is useful to note that the results with uninfected D. melanogaster are similar to prior reports in which higher Ance expression is observed in testes relative to ovaries ( $>1.6$ fold increase in testes; $[31,32])$. However, the Wolbachia infection status in the prior reports is not known.

In crossing experiments, no significant differences are observed in comparisons of the compatible crosses (Table 2 ). However, comparison of the hatch rate resulting from the four incompatible cross types revealed a significantly higher egg hatch rate in crosses of uninfected Ance females and infected wild type males relative to the other incompatible crosses. It is important to note that only heterozygous Ance flies could be used in this experiment. Therefore, only dominant Ance effects would be observed. Furthermore, the possibility cannot be excluded that additional other sub-lethal mutations are co-segregating with the Ance mutation.

A model to describe the observed cross results would require an interaction of Ance with both the rescue and modification mechanisms [33]. Specifically, the Ance/CI model requires that: the Ance mutation in uninfected females results in partially offsetting the Wolbachiainduced modification to sperm, such that a higher frequency of modified sperm are able to escape developmental arrest, resulting in a greater number of viable larvae; and a second Ance/CI interaction occurs in males, which is the origin for the sperm modification. The Ance/CI interaction in males negates the Ance/CI interaction in females, such that the partial offset of $\mathrm{CI}$ is not apparent in crosses between uninfected Ance females and infected Ance males. Since the CI modification and rescue mechanisms and the in vivo Ance substrate are currently unknown [33], this precludes an ability to propose a specific mechanism to explain the above $\mathrm{CI} /$ Ance interaction model. However, observations of Ance expression are not inconsistent with the model proposed above. Specifically, Ance expression differs between Wolbachia-infected and uninfected Drosophila and that the observed variation in expression occurs in opposite directions in female or male flies. The results presented here support additional experiments to test the Ance/CI interaction model.

We note that a broad range of egg hatch rates have been previously reported in Drosophila crosses examining CI, and the egg hatch reported here is at the lower end of the reported range. Egg hatch can be affected by the experimental conditions under which females are held, and the hatch rates observed here are similar to a prior study in which females were made to oviposit in isolation [34].

In addition to Ance, the microarray assay identifies multiple genes that vary in expression level between infected and uninfected cells. In the following text, these are 


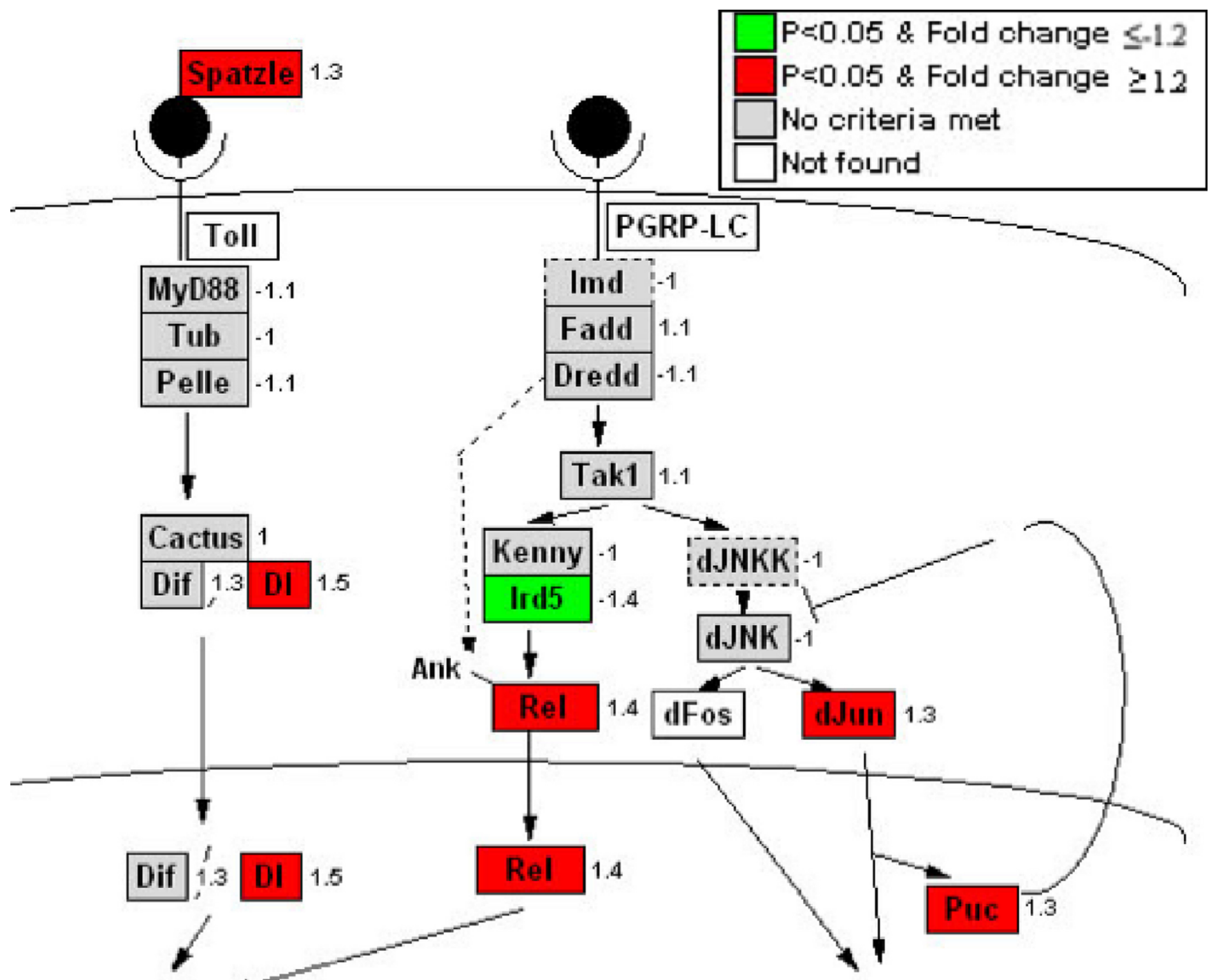

Cytoskeletal related genes

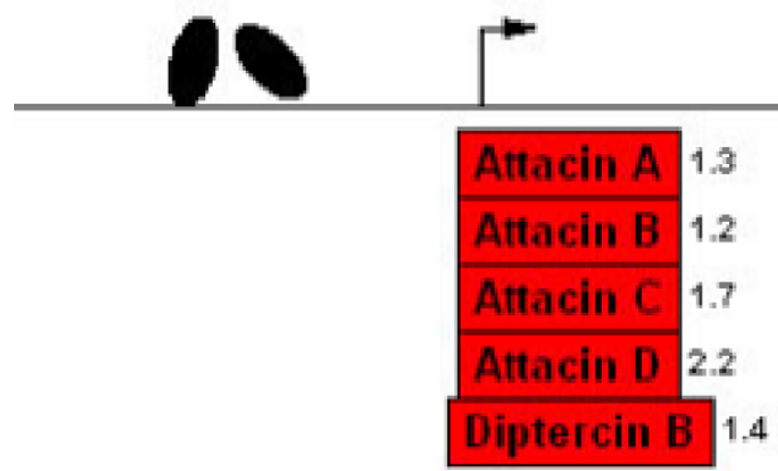

other anti microbi al peptides

\section{Figure 5}

Induction of immune response to Wolbachia infection in S2 cells. Activation of both Toll and Imd pathways result in up-regulation of Dorsal and Relish, which might lead to a specific antimicrobial expression profile against Wolbachia. Negative cross talk between Imd and JNK pathway is also observed [48]. 
Table 3: Classification of differentially expressed genes related to sexual reproduction based on the Gene Ontology (GO) term.

\begin{tabular}{|c|c|c|c|c|c|c|}
\hline \multirow[b]{3}{*}{ Oogenesis } & \multirow{3}{*}{$\begin{array}{l}\text { GO term } \\
\text { Ovarian follicle cell development (sensu Insecta) }\end{array}$} & \multicolumn{5}{|c|}{ Relative Expression Level* } \\
\hline & & \multicolumn{4}{|c|}{ Up } & \multirow{2}{*}{ Down } \\
\hline & & spir & $\operatorname{mip} 130$ & puc & ttk & \\
\hline & & Jra & loco & argos & & sty \\
\hline & Pole plasm assembly (sensu Insecta) & osk & spir & enc & & \\
\hline & Nurse cell to oocyte cell transport (sensu Insecta) & loco & spin & & & jagn \\
\hline & Oocyte dorsal/ventral axis determination & spz & enc & & & \\
\hline & others & gig & & & & sxl \\
\hline \multirow[t]{3}{*}{ Other } & Fertilization & & & & & noi \\
\hline & Germ cell migration & wun & $\bmod (\operatorname{mdg} 4)$ & & & \\
\hline & Male gamete generation & Ance & & & & \\
\hline
\end{tabular}

* Up or down regulated indicates higher or low expression in Wolbachia infected cells, respectively.

grouped based upon their involvement in oogenesis/sex determination, heat shock proteins, and genes involved in immunity.

Wolbachia has been reported to affect host oogenesis [35]. Multiple differentially-expressed genes were observed that have been previously shown to be involved in different processes of oogenesis, a majority of which are related to ovarian follicle cell development (Table 3). The latter is not unexpected due to the critical role of oogenesis in the maternal inheritance of Wolbachia and the somatic stem cell tropism of Wolbachia in Drosophila [36]. Over-expression of $l(2) g l$ and zipper have been previously shown to mimic CI [6], leading a hypothesis that $l(2) g l$ and zipper play a role in the CI mechanism. Consistent with this hypothesis, the results presented here show an up-regulation of $l(2) g l$ in the infected cell.

Additional variation was observed in sex lethal ( $S x l)$, which acts in Drosophila sex determination. Previously, Wolbachia has been shown to suppress sterility in D. melanogaster mutant females with protein-coding lesions in $S x l$ [37]. The results of the prior studies suggested that "Wolbachia does not bypass or reduce the requirement for $S x l$ in the germline in a general way, nor increase overall germline Sxl expression" [37]. Here, microarray data suggests that Wolbachia down-regulates Sxl expression. An additional component in the sex determination/sexual behavior cascade is transformer 2 (Tra-2) [38]. Tra-2 is suppressed $(p<0.05)$ in the infected cell line. However, Tra-2 was not included in Tables 1 or 3 since the observed 1.15 fold reduction does not satisfy designated criteria of $\mathrm{a} \pm 1.2$ fold change.

Multiple transcripts encoding proteins involved in heatshock were observed to be down regulated in Wolbachiainfected S2 cells. A common function of heat shock proteins $(H s p)$ is as molecular chaperones that act to reduce inappropriate inter-protein interaction [39]. Prior studies have shown an ability of heat-shock to abate CI, and distinct $H s p$ isoforms have been reported in comparisons of infected and uninfected Drosophila [40]. Consistent with the prior study, our results indicate a down-regulation of Hsp in Wolbachia-infected cells. Thus, heat-shock may act to negate the impact of reduced Hsp expression in Wolbachia-infected flies.

Wolbachia infection in S2 cells is associated with the induction of antibacterial peptides. Prior studies observed that Wolbachia did not alter the expression of defensin, diptericin and cecropin in D. simulans or A. albopictus [41]. Consistent with the prior results, the microarray assays reported here did not detect changes in the expression of these three antimicrobial peptides. In contrast, Wolbachiainfected cells show higher expression with multiple genes involved in the Toll and Imd immune signaling pathways (Fig. 5). Relish (Rel) and Dorsal $(D l)$ are regulatory proteins in Drosophila that belong to the NF- $\kappa B$ family of transcription factors and play an important role in innate immunity [42]. Drosophila Rel is critical in Imd signaling pathways and is transcriptionally up-regulated in response to gram negative bacteria challenge [43]. An activation of the Toll and Imd immune pathways by gram-negative Wolbachia bacteria could be related to structurally characterized peptidoglycans in Wolbachia [44] that bind to the extracellular peptidoglycan recognition protein (PRGP) and stimulate the Drosophila innate immune signaling pathway [45].

An inhibition of additional expression products involved in immunity is also observed in the microarray assay. The expression of ird5, the Drosophila homolog of $I K K$, is down-regulated. Prior studies show that the activation of Relish requires ird5 [46] and that Escherichia coli survive 100 times better in ird5 mutant lines relative to wild-type Drosophila [47]. Components in the JNK pathway, dJun 
and Puc, were also observed to be higher in infected cells. Prior studies show negative cross talk between the JNK and Imd signaling pathways [48]. Peroxiredoxin 2540 expression was also lower in infected cells. Inhibition of superoxide production has been demonstrated to be important to survival of Anaplasma phagocytophila, which is another intracellular bacterium and close relative to Wolbachia [11]. Together, the latter suggest an ability of Wolbachia to evade the host immune responses.

Eighteen passages after the final shell vial transfection, Wolbachia infection in S2 I could not be detected by PCR (Fig. 1). The disappearance of Wolbachia from the cell is consistent with microarray data showing activation of the host immune response. In previous studies, when Wolbachia was transferred by microinjection from $D$. simulans to D. melanogaster, the latter showed lower densities of Wolbachia than the naturally infected $D$. simulans and did not express high levels of CI [49]. Future experiments should examine the role of immune response in the establishment and maintenance of Wolbachia infection. For example, RNAi technology may be used to interrupt specific immune pathways in S2 cells [19]. The latter cells may then be examined for an ability to sustain Wolbachia infection.

\section{Conclusion}

In summary, the results presented here show the S2 in vitro system to be a useful tool for examining the Wolbachial host interactions that affect host range and CI phenotype and for defining the host immune response to Wolbachia infection and Wolbachia immune evasion mechanisms. Differences observed in the S2 cell culture system are consistent with prior studies examining the Wolbachia interaction within insect hosts. The utility of the in vitro system is further supported by results of the in vivo characterization of Ance expression and CI phenotype assays. The results provide support for the future examination of Ance, Hsp and immune related genes in the Wolbachia/host interaction. The microarray results also provide rationale for examining additional gene products that have not yet been assigned a function, but that are shown to vary between infected and uninfected cells.

\section{Methods}

\section{Drosophila strains and Crosses}

Flies used in this study include Wolbachia-infected and uninfected Drosophila simulans Riverside flies (DSR and DSRT, respectively) and D. melanogaster Canton (DMC and DMCT, respectively). To generate Wolbachia infected Ance flies (named 'AnceW'), male D. melanogaster ('AnceU'; Wolbachia uninfected; genotype: ance [34Eb-2] Adh [D] pr[1] cn[1]/CyO, Adh [nB]; Bloomington Stock Center \#3584) were mated with a Wolbachia-infected D. melanogaster strain with the $\mathrm{CyO}$ balancer chromosome (genotype: wg [Sp-1]/CyO; ry [506] Sb[1] P\{ry [+t7.2] = Delta2-3 \}99B/TM6; Bloomington Stock Center \#2535). Due to maternal inheritance, progeny from the above cross are Wolbachia infected. Subsequently, the infected Ance mutant line is maintained over the $\mathrm{CyO}$ balancer background by selecting for flies with cinnabar eye color and curly wing mutations. All flies were maintained at $25^{\circ} \mathrm{C}$ using standard Drosophila rearing conditions [50].

For the CI assay, one-day-old males were mated with three-day-old females for 12 hours in an apple juice plate container. Females were then isolated individually on a yeast coated apple juice plate to collect eggs. CI was determined as the proportion of hatching eggs, as previous reported [51]. Hatch rate was subjected to arcsine transformation before performing ANOVA.

\section{Establishment of Wolbachia infected $\left(S 2_{1}\right)$ and uninfected $\left(\mathrm{S} \mathbf{U}_{\mathrm{u}}\right)$ cell lines}

Drosophila S2 cells were grown in Schneider's Drosophila medium supplemented with $10 \%$ heat-inactivated fetal bovine serum (Invitrogen). Cells were maintained as described previously $[52,53]$. Wolbachia infection in the S2 cell line was established using a previously described shell vial technique [53]. In brief, DSR eggs were collected by standard procedures [54], homogenized and introduced into S2 cells. To increase the infection level, the shell vial technique was repeated five times sequentially at an interval of every third passage (Fig. 1). The resulting Wolbachiainfected $\mathrm{S} 2$ cell line is subsequently referred to as $\mathrm{S} 2_{\mathrm{I}}$. The uninfected $S 2_{U}$ cell line was generated by dividing $S 2_{I}$ at passage 17 into two sub-lines and tetracycline-treating one sub-line $(10 \mu \mathrm{g} / \mathrm{ml}$ final concentration) [53] as diagrammed in Figure 1.

\section{Microarray analysis}

The $S 2_{\mathrm{I}}$ and $\mathrm{S} 2_{\mathrm{U}}$ cell lines were each divided at passage 23 (Fig. 1) into four independent cultures. Each culture was allowed to grow to confluency and then total RNA was extracted from $5 \times 10^{6}$ Drosophila cells from each of the four replicate preparations using the RNeasy Mini Kit (Qiagen), following the manufacturers instructions. Eight Affymetrix Drosophila genome chips (1.0) were used, with four chips for each of the infected and uninfected treatments. Synthesis of cRNA, labeling, hybridization, staining, washing and detection were performed according to the GeneChip Expression Analysis Technical Manual (Affymetrix). Image data was quantified using the genechip analysis microarray suite 5.0 (MAS 5.0; Affymetrix).

If all eight replicates for a particular probe set were assigned an "absent" value, the probe set was removed from further consideration. The transcript level of the remaining 6,769 probe sets were normalized (divided by the corresponding chip median) and log transformed 
[55]. ANOVA was performed for each probe set in SAS (SAS Institute, Cary, NC) [24]. A Bonferroni significance level was used as an initial criterion for rejecting the null hypothesis of a significant treatment effect $(0.05 / 6769)$. A second arbitrary nominal threshold of $\alpha<0.05$ was used because the Bonferroni correction is overly conservative as tests are correlated [56,57]. A FDR calculation for each p was performed with QVALUE (V1.0) [58]. This threshold $(\mathrm{P}=0.05)$ corresponded to a false discovery rate (FDR) of 0.46 . The change was calculated as the average of four replicates. The following criteria were used to define the differential expression caused by treatment: $\mathrm{P}$ value $<0.05$ and $\geq 1.2$ fold change.

\section{Gene ontology analysis}

MAPPFinder [59] was used to enrich, rank and classify the differentially expressed genes based on Gene Ontology (GO) [60] information of each gene. To visualize the differential expression pattern, before $Z$ value ranking, all gene ontology terms were further filtered manually with the criteria: the number of changed genes: $>2$; the number of measured genes: $>10$; the percent of changed genes: $>5 \%$; the percent of gene presented on the chip: $>20 \%$. If the annotation for interested genes were missed in the gene database of GenMapp, they were examined in Affymetrix NetAffya analysis center [61]. The map for heat shock protein (Hsp) was built by MAPPBuilder [59] based on the information from GO and the Flybase[62]. The map for Drosophila immune response pathway was built by GeneMAPP based on the previous reports $[63,64]$.

\section{Quantitative PCR and quantitative RT-PCR}

Quantitative PCR (qPCR) was performed to characterize the relative Wolbachia infection level in the S2 cell lines and flies. The protocol was similar to prior qPCR amplification using the single-copy $w s p$ and $s u(f k) C$ genes of bacterial and host origin, respectively [65]. S2 cells were quantified using a hemocytometer to obtain $10^{6}$ cells. The S2 cells or DSR females were homogenized in $100 \mu \mathrm{l}$ STE with $0.4 \mathrm{mg} / \mathrm{ml}$ proteinase $\mathrm{K}$ to extract DNA as previously described [66].

For qRT-PCR, RNA extractions were performed on groups of 10 ovaries or 10 testes dissected from one-day post eclosion infected and uninfected Drosophila adults using the RNeasy Mini Kit (Qiagen). DNA contamination was removed with RNase-Free DNase Set (Qiagen). RNA quality and quantity was checked with NanoDrop ND-100 spectrophotometer (NanoDrop Technologies, Inc.). Synthesis of cDNA was performed with Superscript II Reverse Transcriptase (Invitrogen) using specific primer for Ance (AnceQ F 5'-CGGTCACGTTCGATTGGTTG-3' and AnceQ R 5'-CTTCGGTTTCCACGTTGGTTC-3') and Actin gene (ActinQ F 5'-GCTGACCGTATGCAAAAGG-3' and ActinQ R 5'-GCTTGGAGATCCACATCTG-3'). Primers were designed based upon $D$. simulans genbank sequences for Ance and Actin (genbank accession number: NM_057696 and NM_079486, respectively]. qRT-PCR was performed separately with the AnceQ F/R and ActinQ F/R primer pairs using a Miniopticon system (BioRad) with a Platinum SYBR Green qPCR superMix (Invitrogen). qRT-PCR reactions were conducted using a 2 minute step at $50^{\circ} \mathrm{C}$, 2 minute step at $95^{\circ} \mathrm{C}$ and 40 cycles of 15 seconds at $95^{\circ} \mathrm{C}$ and 30 seconds at $56^{\circ} \mathrm{C}$. A fluorescence measurement was made at the end of each cycle. A melting curve analysis was performed at the end of the amplification program to examine for primer-dimers or nonspecific amplification. Assays were performed on two (D. simulans and D. melanogaster wild type) or three (D. melanogaster Ance mutants) independent experiment replicates for each sex and infection type. As an examination for variability, duplicate qRT-PCR reactions were performed for each set of ovaries or testes with both the Ance and Actin primers. Relative expression of Ance gene was calibrated against Actin using the $\Delta \Delta \mathrm{C}_{\mathrm{T}}$ calculation method [67] with:

$$
\Delta \Delta \mathrm{C}_{\mathrm{T}}=\left(\mathrm{C}_{\mathrm{T}, \text { Ance }}-\mathrm{C}_{\mathrm{T}, \text { Actin }}\right)_{\text {infected }}-\left(\mathrm{C}_{\mathrm{T}, \text { Ance }}-\mathrm{C}_{\mathrm{T}, \text { Actin }}\right)_{\mathrm{uninfected}}
$$

$$
\text { Expression variation }=2^{-\Delta \Delta C_{T}}
$$

For comparisons of males and females, the above was modified as follows:

$$
\Delta \Delta \mathrm{C}_{\mathrm{T}}=\left(\mathrm{C}_{\mathrm{T}, \text { Ance }}-\mathrm{C}_{\mathrm{T} \text {,Actin }}\right)_{\text {male }}-\left(\mathrm{C}_{\mathrm{T} \text {,Ance }}-\mathrm{C}_{\mathrm{T}, \text { Actin }}\right)_{\text {female }}
$$

\section{List of Abbreviations used}

Ance, Angiotensin Converting Enzyme; CI, Cytoplasmic Incompatibility; DMC, Drosophila melanogaster Canton; DMCT, Drosophila melanogaster Canton Treated; DSR, Drosophila simulans Riverside; DSRT, Drosophila simulans Riverside Treated; GO, Gene Ontology; qRT-PCR, Quantitative Reverse Transcriptase PCR; T4SS, Type IV Secretion System.

\section{Authors' contributions}

ZX established the Wolbachia infection in Drosophila S2 cell line, prepared the RNA for micorarray assay, performed the gene ontology analysis and drafted the manuscript. LG designed Ance primers, measured the Ance expression by q-RT PCR, and conducted crosses and the related data analysis. YX performed the microarray and CI data analysis. SD conceived of the study, participated in its design and coordination and helped to draft the manuscript. All authors read and approved the final manuscript. 


\section{Additional material}

\section{Additional file 1}

All the Enriched GO term generated from up-regulated genes induced by Wolbachia infection.

Click here for file

[http://www.biomedcentral.com/content/supplementary/14712164-9-1-S1.xls]

\section{Additional file 2}

All the Enriched GO term generated from down-regulated genes induced by Wolbachia infection.

Click here for file

[http://www.biomedcentral.com/content/supplementary/1471-

2164-9-1-S2.xls]

\section{Acknowledgements}

We thank the University of Kentucky for providing a pilot grant to Zhiyong Xi. We acknowledge Kuey-Chu Chen and Donna Wall at the UK Microarray Facility for assistance in conducting microarray assays. We also thank Doug Harrison and Scott O'Neill for providing the D. melanogaster Canton and $D$. simulans strain and Reddy Palli and Xiang Guo for providing comments on this work. This research was supported from NIH grant (NIH-Al51533) to Stephen L. Dobson.

\section{References}

I. Bourtzis K, Miller TA: Insect Symbiosis. Boca Raton, London, New York, Washington, D.C.: CRC Press; 2003.

2. Werren JH, Zhang W, Guo LR: Evolution and phylogeny of Wolbachia: reproductive parasites of arthropods. Proc $R$ Soc Lond $B$ Biol Sci 1995, 26 I(1360):55-63.

3. Sinkins SP: Wolbachia and cytoplasmic incompatibility in mosquitoes. Insect Biochem Mol Biol 2004, 34(7):723-729.

4. Werren JH: Biology of Wolbachia. Annu Rev Entomol 1997, 42:587-609.

5. Masui S, Sasaki T, Ishikawa H: Genes for the type IV secretion system in an intracellular symbiont, Wolbachia, a causative agent of various sexual alterations in arthropods. J Bacteriol 2000, I 82(22):6529-653I.

6. Clark ME, Heath $\mathrm{BD}$, Anderson $\mathrm{CL}$, Karr TL: Induced paternal effects mimic cytoplasmic incompatibility in Drosophila. Genetics 2006, I73(2):727-734.

7. Xi Z, Khoo CC, Dobson SL: Wolbachia establishment and invasion in an Aedes aegypti laboratory population. Science 2005, 3 I 0(5746):326-328.

8. Zabalou S, Riegler M, Theodorakopoulou M, Stauffer C, Savakis C, Bourtzis K: Wolbachia-induced cytoplasmic incompatibility as a means for insect pest population control. Proc Natl Acad Sci USA 2004, I 0 I(42): I 5042-I5045.

9. Hart CA, Winstanley W: What makes a pathogen. Microbiology Today 200I, 28:4-6.

10. Stein M, Rappuoli R, Covacci A: Tyrosine phosphorylation of the Helicobacter pylori CagA antigen after cag-driven host cell translocation. Proc Natl Acad Sci USA 2000, 97: I 263-1268.

II. Carlyon JA, Chan WT, Galan J, Roos D, Fikrig E: Repression of rac2 mRNA expression by Anaplasma phagocytophila is essential to the inhibition of superoxide production and bacterial proliferation. J Immunol 2002, 169:7009-7018.

12. Reynolds KT, Hoffmann AA: Male age, host effects and the weak expression or non-expression of cytoplasmic incompatibility in Drosophila strains infected by maternally transmitted Wolbachia. Genet Res 2002, 80(2):79-87.

13. Veneti Z, Clark ME, Karr TL, Savakis C, Bourtzis K: Heads or tails: host-parasite interactions in the Drosophila-Wolbachia system. Appl Environ Microbiol 2004, 70(9):5366-5372.
14. Schneider I: Cell lines derived from late embryonic stages of Drosophila melanogaster. J Embryol Exp Morphol 1972, 27(2):353-365.

15. Ayres JS, Schneider DS: Genomic dissection of microbial pathogenesis in cultured Drosophila cells. Trends Microbiol 2006, 14(3): $101-104$.

16. Cheng LW, Viala JP, Stuurman N, Wiedemann U, Vale RD, Portnoy DA: Use of RNA interference in Drosophila $S 2$ cells to identify host pathways controlling compartmentalization of an intracellular pathogen. Proc Natl Acad Sci USA 2005, 102(38): | 3646-1365|.

17. Dorer MS, Kirton D, Bader JS, Isberg RR: RNA interference analysis of Legionella in Drosophila cells: exploitation of early secretory apparatus dynamics. PLoS Pathog 2006, 2(4):e34.

18. Elwell C, Engel JN: Drosophila melanogaster S2 cells: a model system to study Chlamydia interaction with host cells. Cell Microbiol 2005, 7(5):725-739.

19. Ramet M, Manfruelli P, Pearson A, Mathey-Prevot B, RA E: Functional genomic analysis of phagocytosis and identification of a Drosophila receptor for $E$. coli. Nature 2002, 4I 6:644-648.

20. Hermans PG, Hart CA, Trees AJ: In vitro activity of antimicrobial agents against the endosymbiont Wolbachia pipientis. J Antimicrob Chemother 200I, 47(5):659-663.

21. Rao R, Well GJ: In vitro effects of antibiotics on Brugia malayi worm survival and reproduction. I Parasitol 2002, 88(3):605-6II.

22. Townson S, Tagboto S, McGarry HF, Egerton GL, Taylor MJ: Onchocerca parasites and Wolbachia endosymbionts: evaluation of a spectrum of antibiotic types for activity against Onchocerca gutturosa in vitro. Filaria $2006,5: 4$.

23. Hurst D, Rylett CM, Isaac RE, Shirras AD: The Drosophila angiotensin-converting enzyme homologue Ance is required for spermiogenesis. Dev Biol 2003, 254(2):238-247.

24. Tatei K, Cai H, Ip YT, Levine M: Race: a Drosophila homologue of the angiotensin converting enzyme. Mech Dev 1995, 5I(2-3): 157-168.

25. Pedra JH, Mclntyre LM, Scharf ME, Pittendrigh BR: Genome-wide transcription profile of field- and laboratory-selected dichlorodiphenyltrichloroethane (DDT)-resistant Drosophila. Proc Natl Acad Sci USA 2004, I 01 ( I 8):7034-7039.

26. Gene Expression Omnibus [http://www.ncbi.nlm.nih.gov/geo]

27. Veneti Z, Clark ME, Zabalou S, Karr TL, Savakis C, Bourtzis K: Cytoplasmic incompatibility and sperm cyst infection in different Drosophila-Wolbachia associations. Genetics 2003 164(2):545-552.

28. Champion de Crespigny FE, Wedell $\mathrm{N}$ : Wolbachia infection reduces sperm competitive ability in an insect. Proc Biol Sci 2006, 273(I593): |455-|458.

29. Clark ME, Veneti Z, Bourtzis K, Karr TL: Wolbachia distribution and cytoplasmic incompatibility during sperm development: the cyst as the basic cellular unit of $\mathrm{Cl}$ expression. Mech Dev 2003, I 20(2): 185-198.

30. Taylor CA, Coates D, Shirras AD: The Acer gene of Drosophila codes for an angiotensin-converting enzyme homologue. Gene 1996, I81(1-2):191-197.

31. Parisi M, Nuttall R, Edwards P, Minor J Naiman D, Lu J, Doctolero M, Vainer M, Chan C, Malley J, Eastman S, Oliver B: A survey of ovary, testis-, and soma-biased gene expression in Drosophila melanogaster adults. Genome Biol 2004, 5(6):R40.

32. Parisi M, Nuttall R, Naiman D, Bouffard G, Malley J, Andrews J, Eastman S, Oliver B: Paucity of genes on the Drosophila $\mathbf{X}$ chromosome showing male-biased expression. Science 2003 299(5607):697-700.

33. Poinsot $D$, Charlat $\mathrm{S}$, Mercot $\mathrm{H}$ : On the mechanism of Wolbachia-induced cytoplasmic incompatibility: confronting the models with the facts. Bioessays 2003, 25(3):259-265

34. Rousset F, Braig HR, O'Neill SL: A stable triple Wolbachia infection in Drosophila with nearly additive incompatibility effects. Heredity 1999, 82(Pt 6):620-627.

35. Dedeine F, Vavre F, Fleury F, Loppin B, Hochberg ME, Bouletreau M: Removing symbiotic Wolbachia bacteria specifically inhibits oogenesis in a parasitic wasp. Proc Natl Acad Sci USA 200I, 98(I I):6247-6252.

36. Frydman HM, Li JM, Robson DN, Wieschaus E: Somatic stem cell niche tropism in Wolbachia. Nature 2006, 44I(7092):509-5I2. 
37. Starr DJ, Cline TW: A host parasite interaction rescues Drosophila oogenesis defects. Nature 2002, 4I8(6893):76-79.

38. Penalva LO, Sanchez L: RNA binding protein sex-lethal (SxI) and control of Drosophila sex determination and dosage compensation. Microbiol Mol Biol Rev 2003, 67(3):343-359.

39. Ang D, Liberek K, Skowyra D, Zylicz M, Georgopoulos C: Biological role and regulation of the universally conserved heat shock proteins. J Biol Chem 1991, 266(36):24233-24236.

40. Feder ME, Karr TL, Yang W, Hoekstra JM, James AC: Interaction of Drosophila and its endosymbiont Wolbachia: natural heat shock and the overcoming of sexual incompatibility. Am Zool 1999, 39:363-373

41. Bourtzis K, Pettigrew MM, O'Neill SL: Wolbachia neither induces nor suppresses transcripts encoding antimicrobial peptides. Insect Mol Biol 2000, 9(6):635-639.

42. Bettencourt R, Asha H, Dearolf C, Ip YT: Hemolymph-dependent and -independent responses in Drosophila immune tissue. Cell Biochem 2004, 92(4):849-863.

43. Dushay MS, Asling B, Hultmark D: Origins of immunity: Relish, a compound Rel-like gene in the antibacterial defense of Drosophila. Proc Natl Acad Sci USA 1996, 93(19): I0343- 10347.

44. Foster J, Ganatra M, Kamal I, Ware J, Makarova K, Ivanova N, Bhattacharyya A, Kapatral V, Kumar S, Posfai J, Vincze T, Ingram J, Moran L, Lapidus A, Omelchenko M, Kyrpides N, Ghedin E, Wang S, Goltsman E, Joukov V, Ostrovskaya O, Tsukerman K, Mazur M, Comb D, Koonin E, Slatko B: The Wolbachia Genome of Brugia malayi: Endosymbiont Evolution within a Human Pathogenic Nematode. PLoS Biology 2005, 3(4): el 2 I.

45. Tanji T, Ip YT: Regulators of the Toll and Imd pathways in the Drosophila innate immune response. Trends Immunol 2005, 26(4): 193-198.

46. Hultmark D: Drosophila immunity: paths and patterns. Curr Opin Immunol 2003, I5(I):12-19.

47. Lu Y, Wu LP, Anderson KV: The antibacterial arm of the Drosophila innate immune response requires an IkappaB kinase. Genes Dev 200I, I5(I): 104-II0.

48. Park JM, Brady H, Ruocco MG, Sun H, Williams D, Lee SJ, Kato T Jr, Richards N, Chan K, Mercurio F, Karin M, Wasserman SA: Targeting of TAKI by the NF-kappa B protein Relish regulates the JNK-mediated immune response in Drosophila. Genes Dev 2004, I 8(5):584-594.

49. Boyle L, O'Neill SL, Robertson HM, Karr TL: Interspecific and intraspecific horizontal transfer of Wolbachia in Drosophila. Science 1993, 260(5 I 15): 1796-1799.

50. Ashburner M: Drosophila, a laboratory handbook. Cold Spring Harbor, N.Y.: Cold Spring Harbor Laboratory; 1989.

5I. Xi Z, Dobson SL: Characterization of Wolbachia transfection efficiency by using microinjection of embryonic cytoplasm and embryo homogenate. Appl Environ Microbiol 2005, 7 I(6):3199-3204.

52. Cherbas L, Cherbas P: Cell culture. Washington, DC Oxford Press; 1998.

53. Dobson SL, Marsland EJ, Veneti Z, Bourtzis K, O'Neill SL: Characterization of Wolbachia host cell range via the in vitro establishment of infections. Appl Environ Microbiol 2002, 68(2):656-660

54. Braig HR, Zhou W, Dobson SL, O'Neill SL: Cloning and characterization of a gene encoding the major surface protein of the bacterial endosymbiont Wolbachia pipientis. J Bacteriol 1998, I 80(9):2373-2378.

55. Quackenbush J: Microarray data normalization and transformation. Nat Genet 2002, 32(Suppl):496-50I.

56. Kerr MK, Churchill GA: Bootstrapping cluster analysis: assessing the reliability of conclusions from microarray experiments. Proc Natl Acad Sci USA 200I, 98(16):896|-8965.

57. Wayne ML, Mclntyre LM: Combining mapping and arraying: An approach to candidate gene identification. Proc Natl Acad Sci USA 2002, 99(23): | 4903- | 4906.

58. Storey JD: A direct approach to false discovery rates. J $R$ Stat Soc Ser B 2002, 64:479-498.

59. Dahlquist KD, Salomonis N, Vranizan K, Lawlor SC, Conklin BR GenMAPP, a new tool for viewing and analyzing microarray data on biological pathways. Nat Genet 2002, 3I(I):19-20.

60. Gene Ontology [http://www.geneontology.org]

61. Affymetrix NetAffya analysis center [http://www.affyme trix.com/analysis/index.affx]
62. Flybase [http://flybase.org/allied-data//k/interactive-fly/aignfam/ heatskfm.htm]

63. Hoffmann JA: The immune response of Drosophila. Nature 2003, 426(6962):33-38.

64. Kim T, Kim YJ: Overview of innate immunity in Drosophila. J Biochem Mol Biol 2005, 38(2): I2I-I 27.

65. McGraw EA, Merritt DJ, Droller JN, O'Neill SL: Wolbachia-mediated sperm modification is dependent on the host genotype in Drosophila. Proc Biol Sci 200I, 268( I 485):2565-2570.

66. O'Neill SL, Giordano R, Colbert AM, Karr TL, Robertson HM: I6S rRNA phylogenetic analysis of the bacterial endosymbionts associated with cytoplasmic incompatibility in insects. Proc Natl Acad Sci USA 1992, 89(7):2699-2702.

67. Livak KJ, Schmittgen TD: Analysis of relative gene expression data using real-time quantitative PCR and the 2(-Delta Delta C(T)) Method. Methods 200I, 25(4):402-408
Publish with Bio Med Central and every scientist can read your work free of charge

"BioMed Central will be the most significant development for disseminating the results of biomedical research in our lifetime. "

Sir Paul Nurse, Cancer Research UK

Your research papers will be:

- available free of charge to the entire biomedical community

- peer reviewed and published immediately upon acceptance

- cited in PubMed and archived on PubMed Central

- yours - you keep the copyright
BiolMedcentral 